\title{
Conductance Switching and Organization of Two Structurally Related Molecular Wires on Gold
}

\author{
Razvan C. Stan, ${ }^{* \dagger}{ }^{\dagger}$ Alexander Kros, ${ }^{\ddagger}$ Namik Akkilic, ${ }^{\S}$ Jeroen Appel, ${ }^{\ddagger}$ and Nusrat J. M. Sanghamitra ${ }^{\dagger}$ \\ ${ }^{\dagger}$ Leiden Institute of Physics-Huygens Laboratories and ${ }^{\ddagger}$ Leiden Institute of Chemistry-Gorlaeus Laboratories, Leiden University, \\ P.O. Box 9504, 2300 RA Leiden, The Netherlands \\ ${ }^{\S}$ Mesa+ Institute for Nanotechnology, University of Twente, P.O. Box 217, 7500 AE Enschede, The Netherlands
}

\section{Supporting Information}

\begin{abstract}
The self-assembly and electron transfer properties of adsorbed organic molecules are of interest for the construction of miniaturized molecular circuitries. We have investigated with scanning probe microscopy the self-organization of two structurally related molecular wires embedded within a supportive alkanethiol matrix. Our results evidence heterogeneous adsorption patterns of the molecular wires on gold with either incommensurate unit cells driven into assembly by lateral interactions or a dynamic, commensurate distribution on gold, along with formation of distinct 2D phases. We also observed diffusion-based conductance switching for one of the molecular wires, due to its propensity toward weaker lateral interactions and $\mathrm{Au}-\mathrm{S}$ adatom formation. We have further demonstrated through the use of scanning tunneling spectroscopy differential current-voltage response for each molecular wire, despite their close structural similarity. Such molecular wires embedded in alkanethiol matrix and exhibiting conductance-switching phenomena have the potential to be used for the functionalization of electrodes in bioelectronic devices.
\end{abstract}

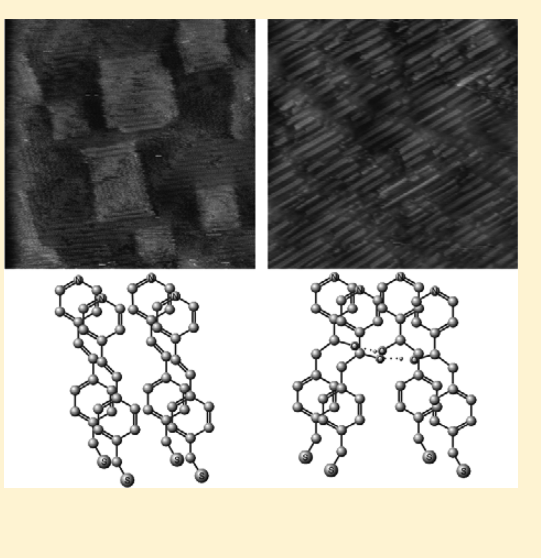

\section{INTRODUCTION}

The self-assembly of organic molecules on metal surfaces renders such simple adsorbates suitable for complementing the silicon-based components of current electronic devices. This is due to their ease and low cost of preparation, their molecular recognition properties, and the modulation of electron transfer to supporting electrodes ("switching" behavior). ${ }^{1,2}$ Because of the industrial use for electrode coating with thin insulating layers and corrosion inhibition properties, the alkanethiol-based self-assembled monolayers (SAM) have been extensively studied. ${ }^{3}$ It has been determined that their insulating properties depend on the length of the alkyl chain and the relative energy positions of the highest occupied and lowest unoccupied molecular orbitals (HOMO-LUMO) band gap as well as on the electrode work function, conformational changes, and molecular orientation. ${ }^{4,5}$ Consequently, in order to enhance the structural quality of the films and their electrical conductivity, aromatic molecules such as OPV (oligo-phenylenevinylenes) are utilized alternatively to form tightly packed, stable monolayers on appropriate surfaces. These OPV have the advantage of increased electrical conductivity when compared to alkanethiols due to $\pi$-electron conjugation paths along the molecular frame and comparatively lower band gap. ${ }^{6}$ However, bulky OPV aromatic rings induce a misfit between the underlying crystalline lattice atoms of the metal electrode and the head groups of the molecular wires, resulting in structural stress such as domain boundaries and dislocation faults. ${ }^{7,8}$ To improve on the quality of the aromatic SAM and to resolve the issue of incommensuration to the metal surface, it has been noted that the introduction of a methylene $\left(-\mathrm{CH}_{2}-\right)$ spacer between the end-thiol group and the aromatic moieties leads to better films in terms of structural integrity but also to pronounced odd-even effects. In the case of the odd-numbered aromatic SAM (where the number of the $-\mathrm{CH}_{2}$ groups is 1,3 , etc.), the greater flexibility around the $\mathrm{C}-\mathrm{S}$ bond translates into less stress imposed on the metal lattice..$^{9-11}$ For the optimal design and fine-tuning of an OPV-based molecular device, we seek to investigate here, at the single-molecule level, their attachment to metal electrodes and shed more light on the intermolecular forces that govern their self-assembly.

In this report, we have used the scanning tunneling microscopy (STM) and conductive-atomic force microscopy (C-AFM) to illustrate the distinctive topography adopted by two structurally related, odd-numbered, aromatic molecular wires (linkers) on $\mathrm{Au}(1,1,1)$ embedded within an alkanethiolbased SAM. We illustrate an interesting differential assembly property and conductance behavior on the gold surface of these linkers having close structural similarity. We also demonstrate the diffusion-based conductance changes that they exhibit and present their current-voltage $(I-V)$ features. Further, we use STM and C-AFM for direct measurement of their electronic properties and establish the involvement of molecular orbitals in mediating electron transfer (ET) to the gold surface. Our

Received: July 16, 2014

Revised: December 22, 2014

Published: December 25, 2014 
results evidence the feasibility of producing mixed SAM of different, finely-tuned chemical and electronic properties.

\section{EXPERIMENTAL SECTION}

Molecular Wires. Synthesis of the linkers is presented in the Supporting Information.

Preparation of Gold Electrodes. Au surfaces were prepared by RF sputtering 250-300 nm onto freshly cleaved V1-grade mica (Jeol B.V.) at a base pressure below $10^{-6}$ bar. Subsequently, annealing with a butane torch was performed so as to obtain flat, recrystallized $\mathrm{Au}$ $(1,1,1)$ surfaces followed by quenching in Milli- $Q$ water.

Sample Preparation. We have used 1-octanethiol to produce selfassembled monolayers (SAM) on the gold surfaces. Both these and the linkers were dissolved in NMP ( $N$-methylpyrrolidone). We have used two incubation methods, based on whether either of the linkers was added together with the alkanethiols (coassembly) or prior to incubation with alkanethiols (preassembly). For the coassembly method the freshly prepared $\mathrm{Au}(1,1,1)$ surfaces were incubated with a mixture of alkanethiols (throughout this study at $1 \mathrm{mM}$ concentration) and linkers (with concentrations ranging from 0.1 to $0.001 \mathrm{mM}$ ) for $22-24 \mathrm{~h}$ at room temperature and washed afterward with NMP. Alternatively, the Au surfaces were first incubated with linkers for $1 \mathrm{~h}$, followed by rinsing with solvent and subsequent addition of alkanethiols and final incubation for another $22 \mathrm{~h}$, also at room temperature.

Ellipsometry. A M2000V variable angle spectroscopic ellipsometer (J.A. Woollam) was used for the thin films' characterization with the incident light at a fixed angle $\left(65^{\circ}\right)$. Data analysis was performed with the WVase software package (J.A. Woollam). The film thickness was calculated assuming a refractive index $n=1.55$.

STM/C-AFM. A PicoSPM I (Agilent Technologies) equipped with a S-scanner and a Digital Instruments multimode microscope (Veeco) with a Nanoscope IIIa controller were used for the scanning tunneling microscopy (STM) work, while a PicoLE microscope (with a current to voltage converter of $1 \mathrm{~V} \mathrm{nA}^{-1}$ sensitivity) was used for conductive atomic force microscopy (C-AFM) measurements. The conductive AFM probes (Cont-E-10, Nanoandmore, Germany) were coated with $\mathrm{Pt}(25 \mathrm{~nm})$ or $\mathrm{Au}(25 \mathrm{~nm}, \mathrm{RF}$ sputtered in house) and $\mathrm{Cr}$ (adhesion layer $\sim 2 \mathrm{~nm}$ ) and had a nominal spring constant of $0.2 \mathrm{~N} / \mathrm{m}$, while mechanically cut Pt/Ir tips (Veeco) were used for STM and STS (scanning tunneling spectroscopy) work. To reduce the water and oxygen content, all conductive measurements were acquired while continuously flushing with $\mathrm{N}_{2}$ in a homemade environmental chamber.

\section{RESULTS AND DISCUSSION}

Distribution of Molecular Wires on AlkanethiolFunctionalized Gold Surfaces. We have investigated the structural and functional properties of two structurally related molecular linkers embedded in alkanethiol SAM. To this end, we have made use of two proximal probe surface techniques: the scanning tunneling microscopy and conductive-atomic force microscopy. STM measurements on pure linker $1\left(\mathrm{~L}_{1}\right)$ or linker $2\left(\mathrm{~L}_{2}\right)$ films on gold have not revealed any ordered structure on the surface, which suggests that the molecular wires are randomly adsorbed. It is likely that upon attachment these linkers adopt a horizontal orientation with respect to the surface, possibly with the benzene rings horizontally $\pi$-stacked, as has been previously observed with other systems. ${ }^{13}$ This result was further corroborated by the ellipsometrical data that indicate a thickness of about $0.4 \pm 0.2 \mathrm{~nm}$ (results not shown). In order to ascertain the presence of the linkers in a standing configuration, use has been made of the 1-octanethiol monolayers $\left(\mathrm{C}_{8}\right)$ to serve as both structural "props" for our molecules and as contrasting background in STM. In contrast to the prevalence of the $\mathrm{C}_{8}$ on the surface, both $\mathrm{L}_{1}$ and $\mathrm{L}_{2}$ were found to be sparsely interspersed within the SAM domains and present in three distinct configurations: as single molecules, as disorganized aggregates, or as ordered domains.

Relatively large $\mathrm{L}_{1}$ domains $(\sim 100 \times 100 \mathrm{~nm})$ were observed on gold terraces, suggestive of robust adsorption at high concentrations $(0.1 \mathrm{mM})$, as presented in Figure 1a. The most common organization of $\mathrm{L}_{1}$ was in the form of narrow, linear arrays, as purported in the stacked arrangement shown in Figure 1b. Such rows were often part of large, nondescript domains containing multiple parallel rows in close proximity or part of larger units resembling a checkerboard. The measured distances between adjacent molecules and between molecular

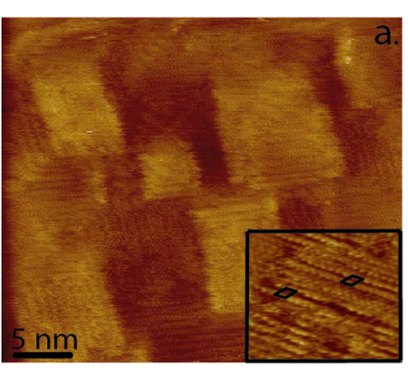

b.
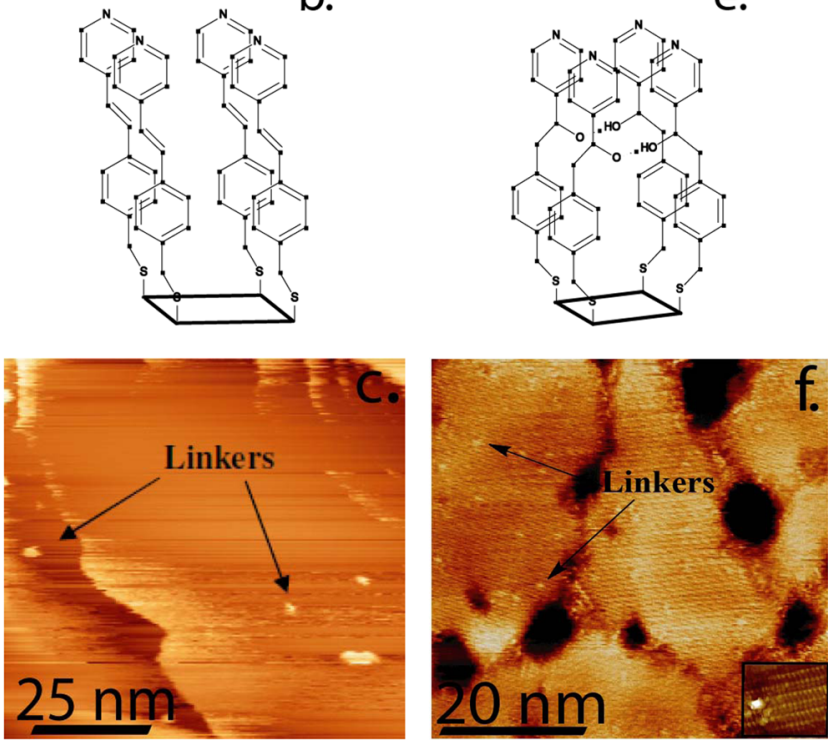

Figure 1. (a) STM height image of extended $\mathrm{L}_{1}$ domains. The thiolprotective acetyl groups were removed during surface functionalization with $\mathrm{NH}_{4} \mathrm{OH}$ or during synthesis by using $\mathrm{LiAlH}_{4}$. Inset $(7 \times 7 \mathrm{~nm})$ presents a high-resolution STM height image of $\mathrm{L}_{1}$ organized in structures consisting of either single or double molecular rows. The basic unit cell is shown as parallelograms. (b) Proposed model of the $\pi$-stacking organization within an individual $L_{1}$ unit cell. (c) C-AFM current image of a mixed SAM prepared by coassembly with 1octanethiols; a multitude of bright (conductive) spots are visible. Image taken at $\sim 4 \mathrm{nN}$ applied force. (d) STM height image of extended $\mathrm{L}_{2}$ domains. Inset $(6 \times 6 \mathrm{~nm})$ presents a high-resolution STM height image of $\mathrm{L}_{2}$ organized in structures consisting mostly of twin molecular rows. The basic unit cell is shown as a parallelogram. (e) Proposed model of the organization of individual $\mathrm{L}_{2}$ cell units. (f) STM height image of a heterogeneous film of $\mathrm{L}_{1}$ and 1-octanethiols. Arrows indicate single molecules; images were obtained in constant current mode. Inset $(7 \times 7 \mathrm{~nm})$ presents a single $\mathrm{L}_{1}$ spot embedded in the alkanethiol matrix, with the relative size of $\sim 3.12 \mathrm{~nm}^{2}$ vs the calculated cross-sectional area of the volume occupied by a single $\mathrm{L}_{1}$ molecule of $1.26 \mathrm{~nm}^{2}$. Tunneling parameters are $I=86 \mathrm{pA}$ and $U=$ $860 \mathrm{mV}$. 
rows are of $0.44 \pm 0.06 \mathrm{~nm}$ and $0.8 \pm 0.1 \mathrm{~nm}$. The $(3 \times \sqrt{ } 3)$ cell is proposed as the basic lattice unit, commensurate to the gold surface, and is shown as parallelograms (inset, Figure 1a).

While single $\mathrm{L}_{2}$ molecules were also found within the $\mathrm{C}_{8}$ domains, most of $\mathrm{L}_{2}$ was present in large scale organizations $\left(\sim 1000-1500 \mathrm{~nm}^{2}\right)$, at high concentrations $(0.1 \mathrm{mM})$, arrayed in single or double molecular rows. Figure $1 \mathrm{~d}$ reveals the distribution of $\mathrm{L}_{2}$ organized in either single rows of molecules or, most dominantly, paired. Figure 1e presents a model of the $\pi$-stacking and $\mathrm{H}$-bond interactions between the molecular linkers assembled on gold. The lattice unit based on the average distances found along the molecular rows of $0.28 \pm 0.03 \mathrm{~nm}$ and between them of $0.29 \pm 0.04 \mathrm{~nm}$ is also presented. The former set of values is consistent with calculated interplanar distances $(0.32 \mathrm{~nm})$ between aromatic moieties. We note here that these values differ from the distances measured for the structurally related $\mathrm{L}_{1}$ and that the packing is much tighter within the $\mathrm{L}_{2}$ structures. The rotational flexibility around the $-\mathrm{CH}_{2}-$ groups, located between the aromatic rings, may contribute to the decrease in the steric hindrance and minimization of the intermolecular distances. This flexibility of $\mathrm{L}_{2}$ molecules contrasts to the rigidity of $\mathrm{L}_{1}$ molecules, brought on by both the rigidity of the coplanar aromatic moieties and the presence of the $\mathrm{C}=\mathrm{C}$ bond.

We have further used C-AFM in order to obtain topographical information related to the linker distribution within the SAM and acquire current-voltage spectra of individual spots. A representative example is shown in Figure 1c, presenting current images of mixed monolayers prepared by coassembly of alkanethiols with the molecular wires (0.01 $\mathrm{mM}$ ). Numerous bright (more conductive) spots of various sizes are noticeable; we assume that these are the physically higher, more conductive linkers (indicated by arrows). Figure 1f presents STM measurements on same type of functionalized surface, where the higher number of conductive features differs from the C-AFM measurements. Despite coadsorbing the alkanethiols with increasing concentrations of $\mathrm{L}_{1}$, the STM images did not show a corresponding increase in number of conductive spots, as previously observed with methylthiolate. ${ }^{14}$ We propose that the reason for this discrepancy lies with both the faster adsorption kinetics of alkanethiols ${ }^{15}$ that will obscure the increase in concentration of the molecular wires and in the difficulty of assessing whether a bright spot observed with STM consists of one or of several clustered linkers.

Irrespective of its co- or preadsorption with the linkers, the gold surface will be restructured as the alkanethiols move from a horizontal organization into a slanted configuration. The linkers have thus limited access to gold adsorption sites and are mainly encountered in the SAM defects or in limited domains on gold (Figure 1a,d) and rarely embedded within the alkanethiol matrix (Figure 1c,f). Because of the steric hindrance by the bulky moieties of the OPV, the adsorption sites will be different than for alkanethiols, in order to accommodate for the lateral chains. ${ }^{16,17} \mathrm{~A}$ recent hypothesis based on grazing incidence X-ray diffraction ${ }^{18}$ has introduced kinetic arguments into the adsorption process. In this model, in a first instance, the adsorption takes place at the "on-top" site while concomitantly the hydrogen atom from the $-\mathrm{SH}$ group is lost; this is followed by a second stage, whereby the chemisorbed sulfur diffuses away either on the gold surface to a more energetically favorable position such as fcc or hcp or together with a gold atom. ${ }^{19}$ This last step will depend on temperature and on the strength of the van der Waals forces and explains the localization of molecules on both the "on-top" sites and/or one of the hollow sites. It has been argued that the strong $\mathrm{Au}-\mathrm{S}$ bond $(74 \mathrm{~kJ} / \mathrm{mol}$ for alkanethiols and $46 \mathrm{~kJ} / \mathrm{mol}$ for arenethiols), together with their different diffusion barriers, is sufficient to perturb the gold lattice and reconstruct its $(22 \times$ $\sqrt{ } 3$ ) surface, with subsequent formation of mono- or biatomic gold pits and the formation of $\mathrm{Au}$ adatom islands.

We emphasize that, within the ordered domains, the general arrangement in pairs of molecular rows and the distances measured across these rows for either $\mathrm{L}_{1}$ or $\mathrm{L}_{2}$ are different than those corresponding to their aromatic moieties horizontally stacked on the surface, as previously determined ${ }^{13}$ (e.g., transstilbene, with a similar structure as $\mathrm{L}_{1}$ ). The calculated distance of $0.65 \mathrm{~nm}$ between the aromatic centers contrasts with the measured average distances of $0.8 \mathrm{~nm}\left(\mathrm{~L}_{1}\right)$ and $0.3 \mathrm{~nm}\left(\mathrm{~L}_{2}\right)$. A study on the assembly of benzylmercaptan, which is structurally similar to both $\mathrm{L}_{1}$ and $\mathrm{L}_{2}$ but for the additional aromatic ring, has revealed orderly domains with intermolecular distances of $0.57 \pm 0.02 \mathrm{~nm}$, commensurable to gold, without any observed flat-lying domains. ${ }^{17}$ We therefore propose that the predominant configuration of the linkers is in a vertical orientation with respect to the surface, either as single-molecules or grouped. This can be of importance for future applications where a pyridine or an imidazole headgroup can coordinate the metal from the redox cavity in protein mutants and thus give direct access to the active center of an enzyme, for increased ET to electrodes ("protein wiring"). ${ }^{31}$

Conductance Switching Events of Single Molecular Wires. In order to further understand the interaction between individual molecular wires with the alkanethiol matrix and the gold surface, we have also measured their dynamic organization for extended intervals. Because the aromatic moieties interact only through van der Waals forces with the alkyl chains, ${ }^{20}$ the electrical conductance of the molecular wires is mainly determined by their structure. The activity of alkanethiolembedded single linkers or clusters thereof can be followed with STM under constant bias and in constant current mode, thus providing time lapses of their adsorption kinetics.

When $\mathrm{L}_{1}$ molecules were imaged, their behavior was marked by disappearance and reappearance in subsequent frames (Figure 2). Under our experimental conditions, removal and reattachment of the molecular wires on the surface was not likely because the switching events were located close to the original spots. This is despite the scanning being performed
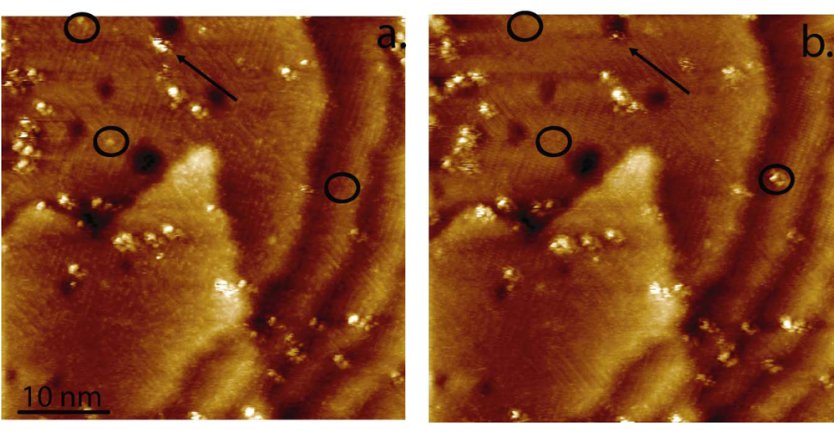

Figure 2. Consecutive STM images of diffusive $\mathrm{L}_{1}$ molecules (bright spots); the circles mark the presence of a conductive spot that is diffusing in either the previous or the subsequent frame. Arrows indicate the change in size of such a conductive spot, caused by the lateral diffusion in the alkanethiol matrix. Tunneling parameters are $U$ $=1.1 \mathrm{~V}$ and $I=20 \mathrm{pA}$; time interval between frames is $2 \mathrm{~min}$. 


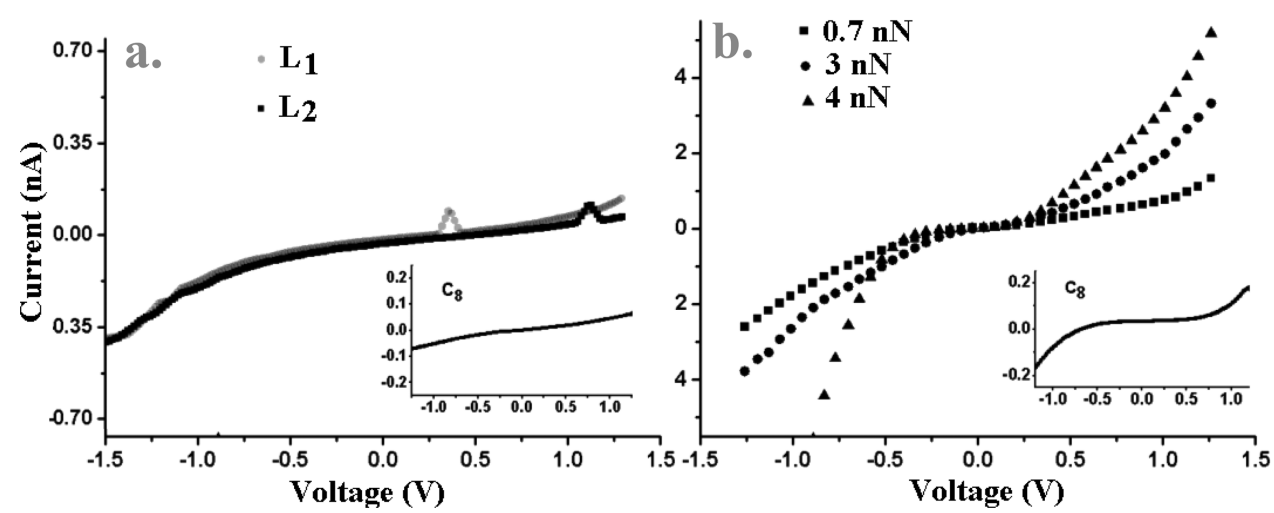

Figure 3. (a) STS $I(V)$ curves (averaged) of $\mathrm{L}_{1}$ and $\mathrm{L}_{2}$. NDR features are evident at different bias ranges. Inset presents averaged $I(V)$ curve obtained over 1-octanethiol domains. Noise in all $I(V)$ curves is $<0.2 \mathrm{pA}$. (b) C-AFM $I(V)$ spectra of various $\mathrm{L}_{1}$ spots at different values of applied forces. NDR features are present over the $0.5-0.7 \mathrm{~V}$ range. The inset presents a typical $I(V)$ response obtained over different 1-octanethiol spots. The nominal spring constants of the C-AFM tips have $10-15 \%$ uncertainty that is reflected in the force values.

from frame to frame across many film defects, where reinsertion could occur. It is however possible that the conductive spots that appear to blink on the surface may represent more than one molecule (Figure 1f).

It is important to note that the conductance switching events were not observed in the case of the structurally similar molecule $L_{2}$, regardless of the sign of the imposed voltage or its localization on the gold surface (Supporting Information). The stability of $\mathrm{L}_{2}$ aggregates on the surface could be enhanced by the energetic contribution brought about by the presence of the polarizable $-\mathrm{OH}$ groups and thus of the extra hydrogenbonding present. We propose that the conductance switching of $\mathrm{L}_{1}$ may be explained by the lateral diffusion within the 1octanethiol matrix and the rearrangement of $\mathrm{L}_{1}$ aggregates. This diffusion process could be due to a combination of thermal energy excitation ${ }^{21}$ and in-plane relaxation of the gold lattice upon thiol adsorption. Our hypothesis stands in contrast with current models for the conductance switching of other OPV, that involve (1) changes in the hybridization state of the $\mathrm{Au}-\mathrm{S}$ bond, ${ }^{22}$ (2) position exchanges on the gold surface defects, ${ }^{23}$ (3) rotations of aromatic moieties, ${ }^{24}$ or (4) the polarizability of the lateral hydroxyl groups upon reversing the sign of applied voltage, $^{25}$ as these cannot account for the differences in behavior that we observed. Because our molecular wires are structural analogues, the conductance switching events should be present in both linkers (models 1,2). Despite the presence of a structural difference between the linkers, i.e., the side hydroxyl group in $\mathrm{L}_{2}$, that also allows for the rotation of the aromatic moieties, this molecular wire does not present conductance switching upon sign reversal of applied bias (models 3, 4).

Conductivity Measurements of Single Molecular Wires. To further unravel the electron transfer properties of $\mathrm{L}_{1}$ and $\mathrm{L}_{2}$, we have performed single-molecule scanning tunneling spectroscopy (STS) and C-AFM $I(V)$ spectroscopy. The spectroscopic data obtained with STM and C-AFM on individual molecules are Presented in Figure 3. An interesting spectroscopic feature for both linkers is the presence of negative differential resistance (NDR). These are present in most $I(V)$ spectra, for the $\mathrm{L}_{1}$ in the range of $0.4-0.5 \mathrm{~V}$ and for $\mathrm{L}_{2}$ at $1.1-1.2 \mathrm{~V}$ (Figure 3a, STS) and $0.65-0.8 \mathrm{~V}$ (Figure $3 \mathrm{~b}$, C-AFM, for each value of applied force). NDR features are not observed in the corresponding $I(V)$ spectra obtained on the alkanethiol film (insets in Figure 3). We also note here that the magnitude of the current measured with STM is lower than the corresponding values obtained by C-AFM, probably due to the insulating air gap that exists between the STM tip and molecules being probed, as opposed to the direct contact between the C-AFM probe and the adsorbates.

In order to model the electron transfer through $\mathrm{L}_{1}$ molecules, it is necessary to consider both the slope in the low-bias regime (ohmic behavior) and the exponential increase in current at higher voltage, while excluding the dielectric breakdown at very high voltages. We have used a modified version of the Simmons equation, ${ }^{26,27}$ in order to account for both the asymmetric molecule $\left(\mathrm{L}_{1}\right)$ and the asymmetry in the tip-substrate junction (i.e., a chemical contact established via the thiol head group and a physical contact made with the STM/C-AFM tip). The dissimilar voltage drop across the molecules can be divided into two components as follows:

$$
V=\alpha V+(1-\alpha V)
$$

where $V$ is the applied bias, $\alpha$ is an asymmetry factor, $\alpha V$ is the elevated tip potential, and $(1-\alpha V)$ the reduced potential of the substrate. The Simmons equation can now be expressed as

$$
\begin{aligned}
I= & \frac{e^{2}}{2 \pi h L^{2}}\left\{(\varphi-\alpha V) \mathrm{e}^{-K(\varphi-\alpha V)^{1 / 2}}-(\varphi+(1+\alpha) V)\right. \\
& \left.\times \mathrm{e}^{-K(\varphi+(1-\alpha) V)^{1 / 2}}\right\}
\end{aligned}
$$

where $I$ is the detected current, $L$ is the barrier width (nm), $A=$ $2(2 m)^{1 / 2} / \hbar$, and $\bar{\varphi}$ is the mean height of the energy gap between the Fermi level and the band edge (HOMO or LUMO). By varying $\alpha$ and the transport parameters of interest (barrier potential and barrier width), we performed a nonlinear square fitting until stable $\chi$-values were achieved. The mean values are presented in Table 1 for the low force $(0.7 \mathrm{nN})$ and high force regime $(4 \mathrm{nN})$. We note that the potential barrier

Table 1. Overview of the Decay Constant $\beta\left(\AA^{-1}\right)$, Tunneling Barrier Height $(\mathrm{nm})$, and Barrier Width $(\mathrm{eV})$ Obtained after Fitting to the Simmons Equation the C-AFM $I(V)$

Measurements on Single $L_{1}$ Molecules

$\begin{array}{cccc}\begin{array}{c}\text { applied force } \\ \text { values }\end{array} & \begin{array}{c}\text { potential barrier } \\ (\mathrm{eV})\end{array} & \begin{array}{c}\text { gap width } \\ (\mathrm{nm})\end{array} & \begin{array}{c}\text { decay constant } \\ \left(\AA^{-1}\right)\end{array} \\ 700 \mathrm{pN} & 3.74 \pm 0.74 & 1.49 \pm 0.08 & 0.69 \pm 0.12 \\ 4 \mathrm{nN} & 6.06 \pm 0.88 & 1.07 \pm 0.17 & 0.51 \pm 0.11\end{array}$


and the tunneling gap widths vary with the increase in applied force, as can be expected. The average decay constant $\beta$ is similar in the high force regime to the one calculated from STM data $\left(0.51 \AA^{-1}\right.$ vs $\left.0.49 \AA^{-1}\right)$ and that both values are smaller than $\beta=0.95 \AA^{-1}$ obtained from $\mathrm{C}_{8}$. The high potential barrier observed at $\sim 4 \mathrm{nN}$ applied force is higher than the value obtained at $\sim 0.8 \mathrm{nN}(6 \mathrm{eV}$ vs $3.74 \mathrm{eV}$ ) which could be due to the higher indentation of the C-AFM tip into the monolayers. It is also possible that the increased compression on $\mathrm{L}_{1}$ may lead to structural denaturation and the shift in the NDR position that we have observed (Figure $3 \mathrm{~b}$ ).

The NDR features in the $I(V)$ spectra were further analyzed in the light of the resonant tunneling through molecular orbitals. Because of the presence of the surrounding alkanethiol matrix, we assume that the trans-cis transitions of the molecular wires are precluded and that the molecule is planar, such that the current steps are not reflective of conformational changes. Reversible NDR peaks were observed when the applied voltages corresponded to energy of the molecular orbitals. ${ }^{28}$ However, the alignment of the Fermi levels of the contact with the energy levels in the molecule is generally unknown, as is the electric field distribution through the junction. ${ }^{29}$ Because of the uninterrupted conjugation system in the $\mathrm{L}_{1}$, as opposed to $\mathrm{L}_{2}$, and the possibility of torsion between the aromatic groups in the latter, the band gap values for these molecules should be different. We have experimentally determined for $L_{1}$ and for $L_{2}$ a band gap of 3.53 and 4.58 $\mathrm{eV}$, respectively, from their optical absorption spectra (Supporting Information). An estimation of the band gap and the pinning of the molecular energy levels that was calculated semiempirically with HyperChem 7 (AM 1) yielded for $L_{1}$ values for $\mathrm{LUMO}=-2.75 \mathrm{eV}, \mathrm{HOMO}=-5.16 \mathrm{eV}$, and a band gap of $2.41 \mathrm{eV}$, while for $\mathrm{L}_{2}$ revealed values for $\mathrm{LUMO}=-0.7$ $\mathrm{eV}, \mathrm{HOMO}=-5.7 \mathrm{eV}$, and a band gap of $5 \mathrm{eV}$. These values contrast to the measurements of the band gap in a solid-state environment or when computed in vacuum and are smaller than the $\sim 7 \mathrm{eV}$ band gap of alkanethiols but closer to the 3.1 $\mathrm{eV}$ value of trans-stilbene with a similar structure to that of $\mathrm{L}_{1}$. ${ }^{30}$ Because the potential profile of the surface is not known and only a potential difference is applied in the ex-situ STM/CAFM setup, we hypothesize that by ramping the voltage, the Fermi level of the gold surface matches the LUMO of the molecular wires and further resonant tunneling can occur. We propose that for the STS data the observed shifts in NDR positions between $L_{1}$ and $L_{2}$ are due to their different band gap that are being probed, as the voltage is ramped. We envision that independent determination, by electrochemical STM or CAFM of the redox potential of these linkers (and thus of their HOMO-LUMO band gaps), can further establish the origin of the observed NDR.

\section{CONCLUSIONS}

We have measured the assembly on gold surfaces and the conductive properties of two structurally analogous molecular wires. We have highlighted the importance of the lateral interactions between OPV and the alkanethiol matrix and put forth a new hypothesis for the conductance switching events that has previously been observed with STM for other OPV. The structural analogues used in this study exhibit subtle rectifying behaviors in their current-voltage responses, allowing for fine-tuning of their electronic properties. Moreover, these molecules have a potential for the modular fabrication of protein biosensors through "click chemistry", as their pyridine functionalities can be used to further attach or detect appropriately engineered proteins. Such sensors can thus potentially benefit from defined orientations and distinct conductive pathways with respect to a metal surface.

\section{ASSOCIATED CONTENT}

\section{Supporting Information}

Synthesis of molecular wires; UV-vis absorption spectra of molecular wires; STM measurements of Linker 2 adsorbates on gold surfaces. This material is available free of charge via the Internet at http://pubs.acs.org.

\section{AUTHOR INFORMATION}

\section{Corresponding Author}

*E-mail strazvan@gmail.com (R.C.S.).

\section{Present Address}

R.C.S.: Institute for Biomedical Sciences, University of São Paulo, CEP 05508-900, São Paulo, Brazil.

\section{Notes}

The authors declare no competing financial interest.

\section{ACKNOWLEDGMENTS}

We thank Profs. T. J. Aartsma, T. H. Oosterkamp, and G. W. Canters for valuable discussions and support and Wim Jesse for assistance with the ellipsometric measurements.

\section{REFERENCES}

(1) Coskun, A.; Spruell, J. M.; Barin, G.; Dichtel, W. R.; Flood, A.; Botros, Y. Y.; Stoddart, J. F. High Hopes: Can Molecular Electronics Realise Its Potential? Chem. Soc. Rev. 2012, 41, 4827-4859.

(2) Aviram, A.; Ratner, M. A. Molecular Rectifiers. Chem. Phys. Lett. 1974, 29, 277-283.

(3) Love, J. C.; Estroff, L. A.; Kriebel, J. K.; Nuzzo, R. G.; Whitesides, G. M. Self-Assembled Monolayers of Thiolates on Metals as a Form of Nanotechnology. Chem. Rev. 2005, 105, 1103-1169.

(4) Wu, H.; Sotthewes, K.; Kumar, A.; Vancso, G. J.; Schön, P.; Zandvliet, H. J. V. Dynamics of Decanethiol Self-Assembled Monolayers on $\mathrm{Au}(111)$ Studied by Time-Resolved STM. Langmuir 2013, 29, 2250-2257.

(5) Nakaya, M.; Shikishima, M.; Shibuta, M.; Hirata, N.; Eguchi, T.; Nakajima, A. Molecular-Scale and Wide-Energy-Range Tunneling Spectroscopy on Self-Assembled Monolayers of Alkanethiol Molecules. ACS Nano 2012, 6, 8728-8734.

(6) Nuzzo, R. G. The Future of Electronics Manufacturing Is Revealed in the Fine Print. Proc. Natl. Acad. Sci. U. S. A. 2005, 98, 4827-4829.

(7) Di Castro, V.; Beccari, M.; Bruni, F.; Caprioli, F.; Decker, F. Comparison of the Protective Effect of Aromatic Thiols Adsorbed on Copper. Surf. Interface Anal. 2010, 42, 601-604.

(8) Kafer, D.; Witte, G.; Cyganik, P.; Terfort, A.; Wöll, Ch. A Comprehensive Study of Self-Assembled Monolayers of Anthracenethiol on Gold: Solvent Effects, Structure, and Stability. J. Am. Chem. Soc. 2006, 128, 1723-1732.

(9) Cyagnik, P.; Buck, M. Competition as a Design Concept: Polymorphism in Self-Assembled Monolayers of Biphenyl-Based Thiols. J. Am. Chem. Soc. 2006, 128, 13868-13878.

(10) Cyagnik, P.; Buck, M.; Azzam, W.; Wöll, Ch. Self-Assembled Monolayers of -Biphenylalkanethiols on $\mathrm{Au}(1,1,1)$ : Influence of Spacer Chain on Molecular Packing. J. Phys. Chem. B 2004, 108, 4989-4996.

(11) Azzam, W.; Cyganik, P.; Witte, G.; Buck, M.; Wöll, Ch. Pronounced Odd-Even Changes in the Molecular Arrangement and Packing Density of Biphenyl-Based Thiol SAMs: A Combined STM and LEED Study. Langmuir 2003, 19, 8262-8270.

(12) Moore, A. M.; Dameron, A. A.; Mantooth, B. A.; Smith, R. K.; Fuchs, D. J.; Ciszek, J. W.; Maya, F.; Yao, Y.; Tour, J. M.; Weiss, P. S. Molecular Engineering and Measurements To Test Hypothesized 
Mechanisms in Single Molecule Conductance Switching. J. Am. Chem. Soc. 2006, 128, 1959-1967.

(13) Tsai, C. S.; Su, C.; Wang, J.; Lin, J. C. STM Study of transStilbene Self-Organized on the Ag/Ge(111) $-(\sqrt{3} \times \sqrt{3}) \mathrm{R} 30^{\circ}$ Surface. Langmuir 2003, 19, 822-829.

(14) De Renzi, V.; Di Felice, R.; Marchetto, D.; Biagi, R.; Pennino, U.; Selloni, A. Ordered $(3 \times 4)$ High-Density Phase of Methylthiolate on $\mathrm{Au}(111)$. J. Phys. Chem. B 2004, 108, 16-20.

(15) Maksymovych, P.; Voznyy, O.; Dougherty, D. B.; Sorescu, D.; Yates, J. T. Gold Adatom as a Key Structural Component in SelfAssembled Monolayers of Organosulfur Molecules on $\mathrm{Au}(1,1,1)$. Prog. Surf. Sci. 2010, 85, 206-240.

(16) Azzam, W.; Bashir, A.; Terfort, T.; Strunskus, T.; Wöll, Ch. Combined STM and FTIR Characterization of Terphenylalkanethiol Monolayers on $\mathrm{Au}(111)$. Effect of Alkyl Chain Length and Deposition Temperature. Langmuir 2006, 22, 3647-3655.

(17) Hallmann, L.; Bashir, A.; Strunskus, T.; Adelung, R.; Staemmler, V.; Wöll, Ch.; Tuczek, F. Self-Assembled Monolayers of Benzylmercaptan and p-Cyanobenzylmercaptan on $\mathrm{Au}(1,1,1)$ Surfaces: Structural and Spectroscopical Characterization. Langmuir 2008, 24, 5726-5733.

(18) Torrelles, X.; Vericat, C.; Vela, M. E.; Fonticelli, M. A.; Millone, D.; Felici, R.; Lee, T. L.; Zegenhagen, J.; Muñoz, G.; Martín-Gago, J. A.; Salvarezza, R. C. Two-Site Adsorption Model for the $(\sqrt{3} \times$ $\sqrt{3}) \mathrm{R} 30^{\circ}$ Dodecanethiolate Lattice on $\mathrm{Au}(111)$ Surfaces. J. Phys. Chem. B 2006, 110, 5586-5594.

(19) Yu, M.; Bovet, N.; Satterley, C. J.; Bengio, S.; Lovelock, K. R. J.; Milligan, P. K.; Jones, R. G.; Woodruff, D. P.; Dhanak, V. True Nature of an Archetypal Self-Assembly System: Mobile Au-Thiolate Species on $\mathrm{Au}(111)$. Phys. Rev. Lett. 2006, 97, 166102.

(20) Donhauser, Z. J.; Mantooth, B. A.; Pearl, T. P.; Kelly, K. F.; Nanayakkara, S. F.; Weiss, P. S. Matrix-Mediated Control of Stochastic Single Molecule Conductance Switching. Jpn. J. Appl. Phys. 2002, 41, 4871-4877.

(21) Wakamatsu, S.; Fujii, S.; Akiba, U.; Fujihira, M. The Dynamical Behavior of a Single Molecule Inserted in a Self-Assembling Monolayer Matrix at Room Temperature. Nanotechnology 2004, 15, 137-41.

(22) Sellers, H.; Ulman, A.; Shnidman, Y.; Eilers, J. E. Structure and Binding of Alkanethiolates on Gold and Silver Surfaces: Implications for Self-Assembled Monolayers. J. Am. Chem. Soc. 1993, 130, 93899401

(23) Moore, A. M.; Mantooth, B. A.; Donhauser, Z. J.; Maya, F.; Price, D. W.; Yao, Y.; Tour, J. M.; Weiss, P. S. Cross-Step PlaceExchange of Oligo(phenylene-ethynylene) Molecules. Nano Lett. 2005, 5, 2292-2297.

(24) Cornil, J.; Karzazi, Y.; Brédas, J. L. Negative Differential Resistance (NDR) in Phenylene Ethynylene Oligomers. J. Am. Chem. Soc. 2002, 124, 3516-3517.

(25) Wu, S. W.; Ogawa, N.; Nazin, G. V.; Ho, W. Conductance Hysteresis and Switching in a Single-Molecule Junction. J. Phys. Chem. C 2008, 112, 5241-5244.

(26) Zhao, J.; Davis, J. J.; Samson, M. P.; Hung, A. Exploring the Electronic and Mechanical Properties of Protein Using Conducting Atomic Force Microscopy. J. Am. Chem. Soc. 2004, 126, 5601-5609.

(27) Wang, W.; Lee, T.; Reid, M. Mechanism of Electron Conduction in Self-Assembled Alkanethiol Monolayer Devices. Phys. Rev. B 2003, 68, 035416.

(28) Scudiero, L.; Barlow, D. E.; Mazur, U.; Hipps, K. W. STM, Orbital-Mediated Tunneling Spectroscopy and UV Photoelectron Spectroscopy of Metal(II) Tetraphenylporphyrins Deposited from Vapor. J. Am. Chem. Soc. 1997, 123, 4073-4080.

(29) McCreery, R. Molecular Electronic Junctions. Chem. Mater. 2004, 16, 4477-4496.

(30) Jiang, C.; Xie, R.; Li, F.; Allen, R. E. Trans-to-Cis Isomerization of Stilbene Following an Ultrafast Laser Pulse. Chem. Phys. Lett. 2009, 474, 263-267.

(31) Jeuken, L.; Vliet, P.; Verbeet, M. Ph.; Camba, R.; McEvoy, J. P.; Armstrong, F. A.; Canters, G. W. Role of the Surface-Exposed and Copper-Coordinating Histidine in Blue Copper Proteins: The
Electron-Transfer and Redox-Coupled Ligand Binding Properties of His117Gly Azurin. J. Am. Chem. Soc. 2000, 122, 12186-12194. 